\title{
Comparison of various antimicrobial agents as catheter lock solutions: preference for ethanol in eradication of coagulase-negative staphylococcal biofilms \\ Correspondence Margaret A. Deighton \\ m_deighton@rmit.edu.au \\ Received 4 September 2008 Accepted 5 January 2009

\author{
Yue Qu, ${ }^{1}$ Taghrid S. Istivan, ${ }^{1}$ Andrew J. Daley, ${ }^{2}$ Duncan A. Rouch ${ }^{1}$ \\ and Margaret A. Deighton ${ }^{1}$ \\ ${ }^{1}$ School of Applied Sciences, RMIT University, Plenty Road, Bundoora, Victoria 3083, Australia
${ }^{2}$ Department of Microbiology, The Royal Children's Hospital, Flemington Road, Parkville, Victoria
3052, Australia
} \\ Coagulase-negative staphylococci (CoNS) are the main causative agents of bacteraemia in infants managed in neonatal intensive care units (NICUs). Intraluminal colonization of long-term central venous catheters by these bacteria and subsequent biofilm formation are the prerequisites of the bloodstream infections acquired in NICUs. The catheter lock technique has been used to treat catheter colonization; however, the optimum choice of antimicrobial agents and their corresponding concentrations and exposure times have not been determined. The effectiveness of catheter lock solutions (CLSs) was assessed by determining the minimal biofilm eradication concentration of antimicrobial agents against CoNS biofilms. Five conventional antibiotics (oxacillin, gentamicin, vancomycin, ciprofloxacin and rifampicin) alone or in combination, as well as ethanol, were evaluated. Ethanol was found to be superior to all of these conventional antibiotics when used as a CLS. A time-kill study and confocal laser scanning microscopy revealed that exposure to $40 \%$ ethanol for $1 \mathrm{~h}$ was sufficient to kill CoNS biofilm cells. To our knowledge, this is the first in vitro study to provide solid evidence to support the rationale of using ethanol at low concentrations for a short time as a CLS, instead of using conventional antibiotics at high concentrations for a long period to treat catheter-related bloodstream infections.
}

\section{INTRODUCTION}

Coagulase-negative staphylococci (CoNS), predominantly Staphylococcus epidermidis, are the most common causative agents of bloodstream infections in neonatal intensive care units (NICUs) (Stoll et al., 2002; Villari et al., 2000). These bacteria are relatively avirulent compared with Staphylococcus aureus and other organisms that also cause bloodstream infections. The pathogenesis of CoNS infection depends mainly on their ability to form biofilms on the surfaces of various polymers (Klingenberg et al., 2005; von Eiff et al., 2002). Long-term catheters are used in both ambulant and hospitalized patients in areas such as intensive care and oncology to provide central venous access for various therapies (Ackoundou-N'guessan et al., 2006; Onland et al., 2006; Opilla et al., 2007; van de Wetering \& van Woensel, 2007). Major adverse effects of

Abbreviations: CLS, catheter lock solution; CLSM, confocal laserscanning microscopy; CLT, catheter lock technique; CoNS, coagulasenegative staphylococci; CRBSI, catheter-related bloodstream infection; MBEC, minimal biofilm eradication concentration; NICU, neonatal intensive care unit; $\mathrm{Pl}$, propidium iodide. long-term catheterization are catheter-related bloodstream infections (CRBSIs), due to intraluminal colonization of catheters by CoNS, and subsequent biofilm formation on the surface of the catheter lumen.

Systemic antibiotics have been widely used to treat CRBSIs, but failures have been reported frequently (Allon, 2004; Benjamin et al., 2001; Berrington \& Gould, 2001; Gagnon et al., 1993) due to the inability of most conventional antibiotic therapies to eradicate biofilm-grown bacteria (Donlan, 2000; Klingenberg et al., 2005) or to poor access of antibiotics to the surface of the catheter lumen (Bastani et al., 2000). These deficits can be overcome by the catheter lock technique (CLT), which involves filling the lumen of the catheter with an antimicrobial agent at high concentration (100-1000 times higher than is used systemically) and allowing the compounds to dwell for a period of time while the catheter is not in use (Messing et al., 1988).

The effectiveness of various antimicrobial agents used as catheter lock solutions (CLSs) to prevent or treat CRBSIs has been compared in numerous studies. Previous studies have shown that the effectiveness of antibiotics is 
suboptimal (Allon, 2003; Curtin et al., 2003; FernandezHidalgo et al., 2006; Kite et al., 2004). In addition, the application of antibiotics may be associated with undesirable effects, such as the development of resistance, allergic reactions and toxicity. In three recent cohort studies, ethanol was found to be extremely effective in the treatment of CRBSIs when applied as a CLS (Metcalf et al., 2004; Onland et al., 2006; Opilla et al., 2007). However, there is still a lack of scientific data on the effectiveness of ethanol in eradication of biofilm-grown bacteria.

Besides the paucity of data on the most effective antimicrobial agents used as CLSs, their optimum concentrations and exposure times also need to be clarified further (Bailey et al., 2002; Berrington \& Gould, 2001). Most investigators have chosen concentrations and exposure time arbitrarily, and hence there is much variation in the effectiveness reported in different studies (Berrington \& Gould, 2001; Lee et al., 2006; Onland et al., 2006; Opilla et al., 2007).

\section{METHODS}

Bacterial isolates and growth conditions. Eight CoNS isolates were used in this study, including two reference strains (RP62a, a biofilm-positive $S$. epidermidis strain, and SP2, a biofilm-negative Staphylococcus hominis strain) and six invasive clinical CoNS isolates from newborns with confirmed CRBSI. These isolates were obtained from blood cultures from infants at the Royal Women's Hospital NICU, Victoria, Australia. Biofilm production of these isolates was examined quantitatively in a previous study by staining the biofilms with Hucker's crystal violet and measuring the $A_{600}$ (Bradford et al., 2006). The isolates were: a biofilm-positive S. epidermidis (isolate no. 3), a biofilm-weak S. epidermidis (no. 4), another biofilm-positive $S$. epidermidis (no. 5) and three Staphylococcus capitis isolates, which only produce biofilms under stress induced by sodium chloride or ethanol (nos 6, 8 and 9). The icaA gene was found in all of the biofilm-positive $S$. epidermidis and $S$. capitis isolates, except for the biofilm-weak isolate S. epidermidis no. 4 (Bradford et al., 2006).

Biofilms were developed in tryptone soy broth (TSB; Oxoid) in 96well microplates as described by Deighton et al. (2001). As S. capitis only produces biofilms when stressed with salt or ethanol, TSB with $4 \%(\mathrm{w} / \mathrm{v})$ sodium chloride was used for biofilm production of $S$. capitis nos 6,8 and 9 .

Antimicrobial agents. Representatives of five different classes of antibiotic and one disinfectant were chosen for the catheter lock experiments: oxacillin, gentamicin, vancomycin, ciprofloxacin, rifampicin (Sigma-Aldrich) and ethanol (Merck). Antibiotic stock solutions were prepared according to the manufacturer's instructions. To compare the effectiveness of single antimicrobial agents in catheter lock experiments, dilutions were prepared with sterile saline, starting from very high concentrations used in the CLT as reported previously (Lee et al., 2006; Mermel et al., 2001; Onland et al., 2006; Opilla et al., 2007; Sherertz et al., 2006). The selected high concentrations were also consistent with published guidelines for the management of CRBSIs (Mermel et al., 2001). The starting concentrations were $10000 \mu \mathrm{g}$ $\mathrm{ml}^{-1}$ for gentamicin and rifampicin, $5000 \mu \mathrm{g} \mathrm{ml}{ }^{-1}$ for oxacillin, vancomycin and ciprofloxacin and $80 \%(\mathrm{v} / \mathrm{v})$ for ethanol. These concentrations are defined as pharmacological concentrations at which no apparent precipitation of antibiotic powder or evaporation of solutions occurs.
The double or triple combinations of conventional antibiotics were chosen based on their reported effectiveness against CoNS biofilms (Saginur et al., 2006). Therapeutic concentrations were used for individual antibiotics in combinations that have been defined as the Clinical and Laboratory Standards Institute breakpoints for determining resistance (Table 1).

In vitro model to predict the efficacy of CLSs. The efficacy of single antibiotics, antibiotic combinations and ethanol against CoNS biofilms was examined. Preformed $24 \mathrm{~h}$ biofilms were exposed to different concentrations and combinations of antimicrobial agents in saline for $24 \mathrm{~h}$ as follow. Two hundred microlitres of single agents at concentrations ranging from $4 \mu \mathrm{g} \mathrm{ml}^{-1}$ to 5000 or $10000 \mu \mathrm{g} \mathrm{ml} \mathrm{g}^{-1}$, double or triple antibiotic combinations at therapeutic concentrations (Table 1) or ethanol (10-80\%) were added to the biofilms. After $24 \mathrm{~h}$ of exposure, the biofilms were washed three times with saline to remove any residual antimicrobial agents. Sterile resin beads $(0.2 \mathrm{~g}$ per well, Amberlite XAD-16; Rohm and Haas) were then added to minimize the chance of antibiotic carryover. TSB $(200 \mu \mathrm{l})$ was then added to each well and microplates were incubated at $37^{\circ} \mathrm{C}$ for a further $48 \mathrm{~h}$. A microplate shaker (speed 2, Titretek; Flow Laboratories) was used to facilitate the multiplication and release of any living cells remaining in the biofilms. After $48 \mathrm{~h}, 150 \mu \mathrm{l}$ of the contents in each well of the microplate was transferred to a $\mathrm{U}$ bottomed microplate and examined visually for turbidity. The lowest concentration of antimicrobial agent corresponding to clear wells was defined as the minimal biofilm eradication concentration (MBEC) for successful use in the CLT. This concentration is generally higher than the MIC for planktonic cultures and also higher than the minimum biofilm inhibitory concentration for biofilms, and targets complete killing of biofilm-embedded cells. This method was defined as the broth recovery method, in contrast to the plate recovery method, which employs agar plates to recover the remaining living cells after treatment.

Optimum concentration and duration of ethanol exposure for catheter lock applications. Two hundred microlitres of ethanol at concentrations ranging from 10 to $80 \%$ was added to $24 \mathrm{~h}$ CoNS biofilms and allowed to remain in contact for $1 \mathrm{~min}, 15 \mathrm{~min}, 1 \mathrm{~h}, 4 \mathrm{~h}$, $8 \mathrm{~h}$ or $24 \mathrm{~h}$. After the specified contact time, the MBEC of ethanol was determined as described above, but without adding resin beads to the wells. The ethanol-treated biofilms of RP62a and SP2 in the original microplate were then collected with sterile cotton-tipped

Table 1. Antibiotic combinations and the test concentrations of individual agents

\begin{tabular}{|lc|}
\hline Combination & Therapeutic concentrations $\left(\boldsymbol{\mu} \mathbf{~ m l}^{-\mathbf{1}}\right) \dagger$ \\
\hline $\mathrm{G}: \mathrm{R}$ & $16: 4$ \\
$\mathrm{O}: \mathrm{R}$ & $0.5: 4$ \\
$\mathrm{~V}: \mathrm{R}$ & $32: 4$ \\
$\mathrm{C}: \mathrm{R}$ & $4: 4$ \\
$\mathrm{G}: \mathrm{O}: \mathrm{R}$ & $16: 0.5: 4$ \\
$\mathrm{G}: \mathrm{V}: \mathrm{R}$ & $16: 32: 4$ \\
$\mathrm{G}: \mathrm{C}: \mathrm{R}$ & $16: 4: 4$ \\
$\mathrm{O}: \mathrm{V}: \mathrm{R}$ & $0.5: 32: 4$ \\
$\mathrm{O}: \mathrm{C}: \mathrm{R}$ & $0.5: 4: 4$ \\
$\mathrm{~V}: \mathrm{C}: \mathrm{R}$ & $32: 4: 4$ \\
& \\
\hline
\end{tabular}

${ }^{\star} \mathrm{G}$, Gentamicin; O, oxacillin; V, vancomycin; C, ciprofloxacin; R, rifampicin.

$\dagger \mathrm{G}: \mathrm{R}$ ratio, for example, of $16: 4$ represents $16 \mu \mathrm{g}$ gentamicin $\mathrm{ml}^{-1}$ plus $4 \mu \mathrm{g}$ rifampicin $\mathrm{ml}^{-1}$. 
swabs and cultured on nutrient agar plates to detect any viable cells embedded in the biofilms that could not be detected by the broth recovery method.

Confocal laser-scanning microscopy (CLSM). Sterile polystyrene pieces $(\sim 0.5 \times 0.5 \mathrm{~cm})$ were aseptically cut from the bottom of a 24 well microplate with extreme caution to avoid any scratch to the tissue culture treated surface. The polystyrene pieces were then transferred to a microwell containing an S. epidermidis RP62a bacterial suspension of $\sim 10^{7}$ c.f.u. $\mathrm{ml}^{-1}$ and incubated overnight at $37{ }^{\circ} \mathrm{C}$. The pieces, with biofilm, were then rinsed three times with $0.9 \%$ saline to remove planktonic bacteria. One of the following treatments was applied to the $S$. epidermidis RP62a biofilms grown on the polystyrene pieces: (i) $0.9 \%$ saline for $24 \mathrm{~h}$, as an untreated biofilm control; (ii) $5000 \mu \mathrm{g}$ oxacillin $\mathrm{ml}^{-1}$ for $24 \mathrm{~h}$; (iii) $5000 \mu \mathrm{g}$ vancomycin $\mathrm{ml}^{-1}$ for $24 \mathrm{~h}$; (iv) $256 \mu$ g ciprofloxacin $\mathrm{ml}^{-1}$ for $24 \mathrm{~h}$; (v) $40 \%$ ethanol for $1 \mathrm{~h}$; or (vi) $40 \%$ ethanol for $4 \mathrm{~h}$. The treated biofilms were then stained with a LIVE/DEAD BacLight viability kit, containing $3.35 \mu \mathrm{M}$ SYTO-9 and $20 \mu \mathrm{M}$ propidium iodide (PI), at $22{ }^{\circ} \mathrm{C}$ for $15 \mathrm{~min}$ in the dark. The fluorescently stained polystyrene pieces were then washed twice with $0.9 \%$ saline. The viability of biofilm cells was examined immediately using a Zeiss LSM $510 \mathrm{~Pa}$ confocal laser-scanning microscope with Zeiss Axioplan upright microscopes (Leica Microsystems). To minimize artefacts associated with simultaneous dual wavelength excitation, all samples were sequentially scanned, frame-by-frame, first at $488 \mathrm{~nm}$ and then at $561 \mathrm{~nm}$. A $\times 63$ oil objective was used in all imaging experiments.

Data analysis. All experiments were run in duplicate and repeated at least three times. A fourth duplicate was performed if the results were inconsistent.

\section{RESULTS AND DISCUSSION}

\section{Comparison of the effectiveness of antibiotics and ethanol as a CLS}

Ethanol was found to be the most efficient of the antimicrobial agents examined for eradication of the CoNS biofilms after $24 \mathrm{~h}$ exposure. A low concentration of $20 \%$ ethanol killed all cells embedded in the biofilms after overnight exposure. Gentamicin, oxacillin and vancomycin, even at very high pharmacological concentrations $\left(10000,5000\right.$ and $5000 \mu \mathrm{g} \mathrm{ml}^{-1}$, respectively), failed to eradicate the biofilm cells of most CoNS isolates. The only exceptions were the biofilms formed by S. epidermidis nos 3 and 4, which responded to gentamicin. In addition, ciprofloxacin and rifampicin achieved biofilm killing of all isolates when exposure lasted for $24 \mathrm{~h}$, but only at relatively high concentrations (32-128 and 256-512 $\mu \mathrm{g} \mathrm{ml} \mathrm{m}^{-1}$, respectively) (Table 2).

The inability of conventional antibiotics commonly used in NICUs (gentamicin, oxacillin and vancomycin) at very high concentrations to kill all biofilm cells indicates their unsuitability as a CLS. This in vitro finding is supported by frequent reports of failure of CRSBIs to respond to oxacillin, gentamicin, vancomycin or teicoplanin in the absence of catheter removal (Allon, 2004; Castagnola et al., 2006; Fernandez-Hidalgo et al., 2006; Guedon et al., 2002). Although vancomycin and teicoplanin lock solutions have been found to be effective in the prevention of catheterrelated infections (van de Wetering \& van Woensel, 2007), their effectiveness on established CoNS biofilms was found to be poor in this study. Ciprofloxacin and rifampicin were able to completely kill biofilm-embedded cells. However, the value of ciprofloxacin and rifampicin in biofilm killing is limited by the high doses and related expense and drug toxicity, and the ease with which rifampicin induces resistance in bacterial populations (Dunne et al., 1993). Our findings were consistent with a recent study by Lee et al. (2006), which found that ciprofloxacin (1000$\left.5000 \mu \mathrm{g} \mathrm{ml}^{-1}\right)$ and rifampicin $\left(5000 \mu \mathrm{g} \mathrm{ml}^{-1}\right)$ completely eradicated staphylococcal biofilms after a short-term exposure, in contrast to vancomycin $\left(5000 \mu \mathrm{g} \mathrm{ml}^{-1}\right)$ and gentamicin $\left(10000 \mu \mathrm{g} \mathrm{ml} \mathrm{m}^{-1}\right)$, which were much less effective, even after long-term exposure (Lee et al., 2006). Differences in the MBEC values of single antibiotics reported in our study and the study by Lee et al. (2006) could be due to the different diluents used for preparing the CLS or the different ages of the biofilms. Sterile saline was used in our study as the test medium to mimic the clinical situation, whilst TSB, a rich medium for bacterial growth, was used in the study by Lee et al. (2006). We examined the effect of antimicrobial agents on $24 \mathrm{~h}$

Table 2. MBECs of single antimicrobial agents against $24 \mathrm{~h}$ CoNS biofilms

Values are geometric means of at least three duplicates. Variation between three duplicates was less than fourfold in all instances.

\begin{tabular}{|c|c|c|c|c|c|c|}
\hline Strain identity & $\begin{array}{c}\text { Gentamicin } \\
\left(\mu \mathrm{g} \mathrm{ml}^{-1}\right)\end{array}$ & $\begin{array}{l}\text { Oxacillin } \\
\left(\mu \mathrm{g} \mathrm{ml}^{-1}\right)\end{array}$ & $\begin{array}{c}\text { Vancomycin } \\
\left(\mu \mathrm{g} \mathrm{ml}^{-1}\right)\end{array}$ & $\begin{array}{l}\text { Ciprofloxacin } \\
\left(\mu \mathrm{g} \mathrm{ml}^{-1}\right)\end{array}$ & $\begin{array}{c}\text { Rifampicin } \\
\left(\mu \mathrm{g} \mathrm{ml}^{-1}\right)\end{array}$ & Ethanol (\%) \\
\hline S. epidermidis RP62a & $>10000$ & $>5000$ & $>5000$ & 64 & 512 & 20 \\
\hline S. hominis SP2 & $>10000$ & 5000 & $>5000$ & 32 & 512 & 20 \\
\hline S. epidermidis no. 3 & 128 & $>5000$ & $>5000$ & 64 & 512 & 20 \\
\hline S. epidermidis no. 4 & 8 & $>5000$ & $>5000$ & 128 & 256 & 20 \\
\hline S. epidermidis no. 5 & $>10000$ & $>5000$ & $>5000$ & 128 & 512 & 20 \\
\hline S. capitis no. 6 & $>10000$ & $>5000$ & 5000 & 64 & 512 & 20 \\
\hline S. capitis no. 8 & $>10000$ & $>5000$ & 5000 & 128 & 512 & 20 \\
\hline S. capitis no. 9 & $>10000$ & $>5000$ & 5000 & 64 & 512 & 20 \\
\hline
\end{tabular}


biofilms, whilst Lee et al. (2006) used 5-day biofilms, which could be expected to behave differently.

Interestingly, there were no significant differences found in the present study in the MBECs of single antimicrobial agents for strains with different biofilm-forming abilities. The concentrations of these agents required to eradicate the adherent cells of the biofilm-negative S. hominis SP2 were the same as those required for S. epidermidis RP62a biofilms, although the latter formed much thicker biofilms than the former (Table 2). Although classified as a biofilmnegative strain, SP2 does form an adherent monolayer on various biomaterials (Christensen et al., 1985). It is therefore likely that the biofilm mode of growth, rather than the ability to form dense biofilm, leads to its resistance to antimicrobial agents. A parallel study by us comparing the antimicrobial susceptibilities of biofilms, adherent monolayers and planktonic cells at similar densities reported the same conclusion when targeting $99.99 \%$ biofilm bacterial killing (unpublished data). Similar results were also found in a recent study, in which a biofilmnegative mutant strain, M187sn3, had the same or an even higher resistance level to most antibiotic locks as its biofilm-positive ancestor (Edmiston et al., 2006).

At therapeutic concentrations, most combinations failed to kill the biofilm cells, indicating the ineffectiveness of biofilm killing of antibiotic combinations at achievable serum concentrations (Table 3). Combinations containing gentamicin were effective against biofilms of $S$. epidermidis no. 4, unless they contained oxacillin (Table 3). Vancomycin combined with rifampicin has been suggested for the treatment of $S$. epidermidis biofilm-associated infections (Monzon et al., 2001; Shama et al., 2002), but our study found no advantage of this combination over rifampicin alone in biofilm eradication. Vancomycin may, however, minimize the risk of rifampicin resistance developing (Zimmerli et al., 1994). Besides the suboptimal effectiveness of conventional antibiotics, the risk of developing resistance, the high cost of antibiotic usage and the possibility of the accidental infusion of highly concentrated solutions into the circulation during the lock procedure and related toxicity cannot be ignored.

\section{Optimum concentration of ethanol and duration of treatment for catheter locks}

Biofilm cells of all isolates were killed within $1 \mathrm{~min}$ of exposure to $60-80 \%$ ethanol and in $1 \mathrm{~h}$ of exposure to $40 \%$ ethanol. Exposure for $>1 \mathrm{~h}$ was required only for $20 \%$ ethanol and no further advantage was achieved by increasing the dwell time beyond $4 \mathrm{~h}$ (Fig. 1).

In agreement with Sherertz et al. (2006), we showed that ethanol eradicates biofilm bacteria much more effectively than commonly used antibiotics if used as a CLS. Moreover, the use of ethanol for the CLT is simpler and easier than previously recommended antibiotic regimens. We also extended these findings further by showing that exposure of CoNS biofilms to $40 \%$ ethanol for $1 \mathrm{~h}$ was sufficient to eradicate the biofilm bacteria. In addition, low concentrations of ethanol (25-60\%) do not cause catheter occlusion (Laird et al., 2005) or degradation of the catheter biomaterial (Opilla et al., 2007), and ethanol is compatible with heparin and EDTA, the anticoagulant agents commonly used in the CLT (Ackoundou-N'guessan et al., 2006; Raad et al., 2007). A shorter dwell time also reduces the risk of spillage of ethanol into the circulation and its associated toxicity (Ackoundou-N'guessan et al., 2006). We therefore suggest that this concentration and exposure time is optimum for clinical use.

The superiority of ethanol over conventional antibiotics can be explained by its hydrophilic nature and small size, which enable it to obtain access to deep biofilm cells, as the biofilm matrix is highly hydrated. This is in contrast to other antimicrobial agents that may have restricted diffusion gradients through the biofilm matrix. Although it has been suggested that 1-2 mm thick biofilms may have a diffusion delay for ethanol to pass through the dense biofilm structure, no solid evidence has been presented (Sissons et al., 1996). In addition, as ethanol has extremely high bactericidal activity against planktonic cells, it could also be effective in killing the 'less active' cells in biofilms, i.e. the cells presenting biofilmspecific genes or the more tolerant 'persister' cells.

Our CLSM study revealed that exposure to $40 \%$ ethanol for $1-4 \mathrm{~h}$, or $256 \mu \mathrm{g}$ ciprofloxacin $\mathrm{ml}^{-1}$ for $24 \mathrm{~h}$, worked

Table 3. Regrowth of biofilm bacteria after exposure to double and triple combinations of antibiotics at therapeutic concentrations

For abbreviations and therapeutic concentrations of individual antibiotics, see Table 1. +, Regrowth; -, no regrowth.

\begin{tabular}{|c|c|c|c|c|c|c|c|c|c|c|}
\hline Strain identity & $R: G$ & $\mathrm{R}: \mathrm{V}$ & $\mathrm{R}: \mathrm{O}$ & $\mathrm{R}: \mathrm{C}$ & $\mathrm{R}: \mathrm{G}: \mathrm{O}$ & $R: G: V$ & $R: G: C$ & $\mathrm{R}: \mathrm{O}: \mathrm{V}$ & $\mathrm{R}: \mathrm{O}: \mathrm{C}$ & $\mathrm{R}: \mathrm{V}: \mathrm{C}$ \\
\hline S. hominis $\mathrm{Sp} 2$ & + & + & + & + & + & + & + & + & + & + \\
\hline S. epidermidis no. 4 & - & + & + & + & + & - & - & + & + & + \\
\hline S. epidermidis no. 5 & + & + & + & + & + & + & + & + & + & + \\
\hline S. capitis no. 6 & + & + & + & + & + & + & + & + & + & + \\
\hline
\end{tabular}




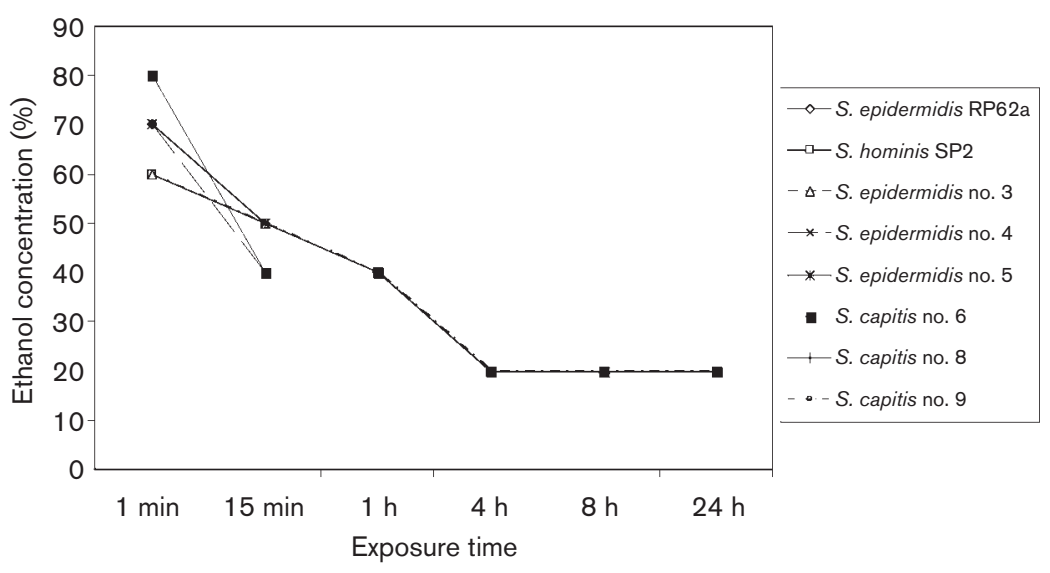

Fig. 1. Concentrations and exposure times of ethanol required to kill all CoNS residing in biofilms. Experiments were repeated in duplicate three times and the data were identical. most efficiently in biofilm cell killing (Fig. 2d-f). Exposure to first-line conventional antibiotics, such as oxacillin and vancomycin, at very high concentrations only partially killed the biofilm cells (Fig. 2b, c).

CLSM in combination with a LIVE/DEAD BacLight viability kit has been used successfully to examine the efficacy of antimicrobial agents on S. epidermidis biofilm eradication (Qin et al., 2007a, b). The LIVE/DEAD BacLight viability kit is a fluorescence-based stain for determining bacterial cell viability and contains two components. SYTO-9 is a membrane-permeant nucleic acid-labelling dye that labels all cells and fluoresces green. The second dye, PI, is another nucleic acid-labelling dye that only enters cells with compromised or damaged cytoplasmic membranes. PI has been reported to have a stronger affinity for nucleic acids, competing with and quenching the SYTO-9 and then fluorescing red (Stocks, 2004). According to the manufacturer, once stained with the LIVE/DEAD BacLight viability kit, the dead cells in a bacterial population present as a bright red colour, whilst live cells are a bright green colour, leaving the background non-fluorescent. This theory is consistent with our results when cell-wall-attacking antibiotics, such as oxacillin and vancomycin, were tested (Fig. 2b, c). However, when antimicrobial agents with intracellular targets or more complex killing mechanisms were tested in our study, the dead cells presented as a reduced green colour or displayed an orange colour, indicating that only partial quenching of SYTO-9 by PI or simultaneous staining of cells by PI and SYTO-9 had occurred. Similar results were obtained in a previous study by Berney et al. (2007). These authors found that Escherichia coli with an intact outer membrane showed strong green fluorescence intensity and those cells with cytoplasmic membrane damage displayed a decreased green fluorescence intensity. An incomplete replacement and quenching of SYTO-9 fluorescence by PI has been proposed to explain this phenomenon (Berney et al., 2007). Based on the report by Berney et al. (2007) as well as the current study, reduction of green fluorescence density, but not the absolute absence of green signal, should be used as indicators of bacterial killing when the BacLight kit is used to examine viability. This theory is more significant when antimicrobial agents with intracellular activity rather than cell-wall-active agents are tested, i.e. ciprofloxacin and ethanol. The incomplete replacement could be explained by the poor effect of these antimicrobial agents on membrane integrity in Staphylococcus spp. (O'Neill et al., 2004), the much lower fluorescent intensity of PI compared with SYTO9 $(8: 300)$ when excited (Stocks, 2004) or the presence of viable but non-culturable cells in the biofilms after antimicrobial exposure (Alam et al., 2007).

Concerning the methodology, the definition of successful catheter lock application used in the present study was the complete absence of recoverable living cells following in vitro exposure. Successful treatment using the catheter lock technique has been defined as negative blood cultures and resolution of fever a few days after completion of a 3-week treatment course (Allon, 2004). However, this definition has not been validated by in vitro studies. Unless complete eradication of biofilm cells is achieved, the remaining living cells will rebuild the biofilm once antimicrobial stress diminishes, as host immune mechanisms can neither reach the lumen of catheters nor eradicate any remaining living cells in biofilms (Lewis, 2005; Spoering \& Lewis, 2001).

Currently, there is no universally accepted in vitro model of catheter-related biofilm infection. The model of biofilm formation and antimicrobial treatment used in our study was intended to replicate the clinical situation as closely as possible. Biofilm development and treatment were carried

Fig. 2. Biofilms of $S$. epidermidis RP62A treated with antimicrobial agents and then stained with SYTO-9 (bright green for live cells) and PI (red or orange, or loss of bright green for dead cells). (a) Untreated biofilm control; (b) biofilm treated with $5000 \mu \mathrm{g}$ oxacillin $\mathrm{ml}^{-1}$ for $24 \mathrm{~h}$; (c) biofilm treated with $5000 \mu \mathrm{g}$ vancomycin $\mathrm{ml}^{-1}$ for $24 \mathrm{~h}$; (d) biofilm treated with $256 \mu \mathrm{g}$ ciprofloxacin $\mathrm{ml}^{-1}$ for $24 \mathrm{~h}$; (e) biofilm treated with $40 \%$ ethanol for $1 \mathrm{~h}$; and (f) biofilm treated with $40 \%$ ethanol for $4 \mathrm{~h}$. 

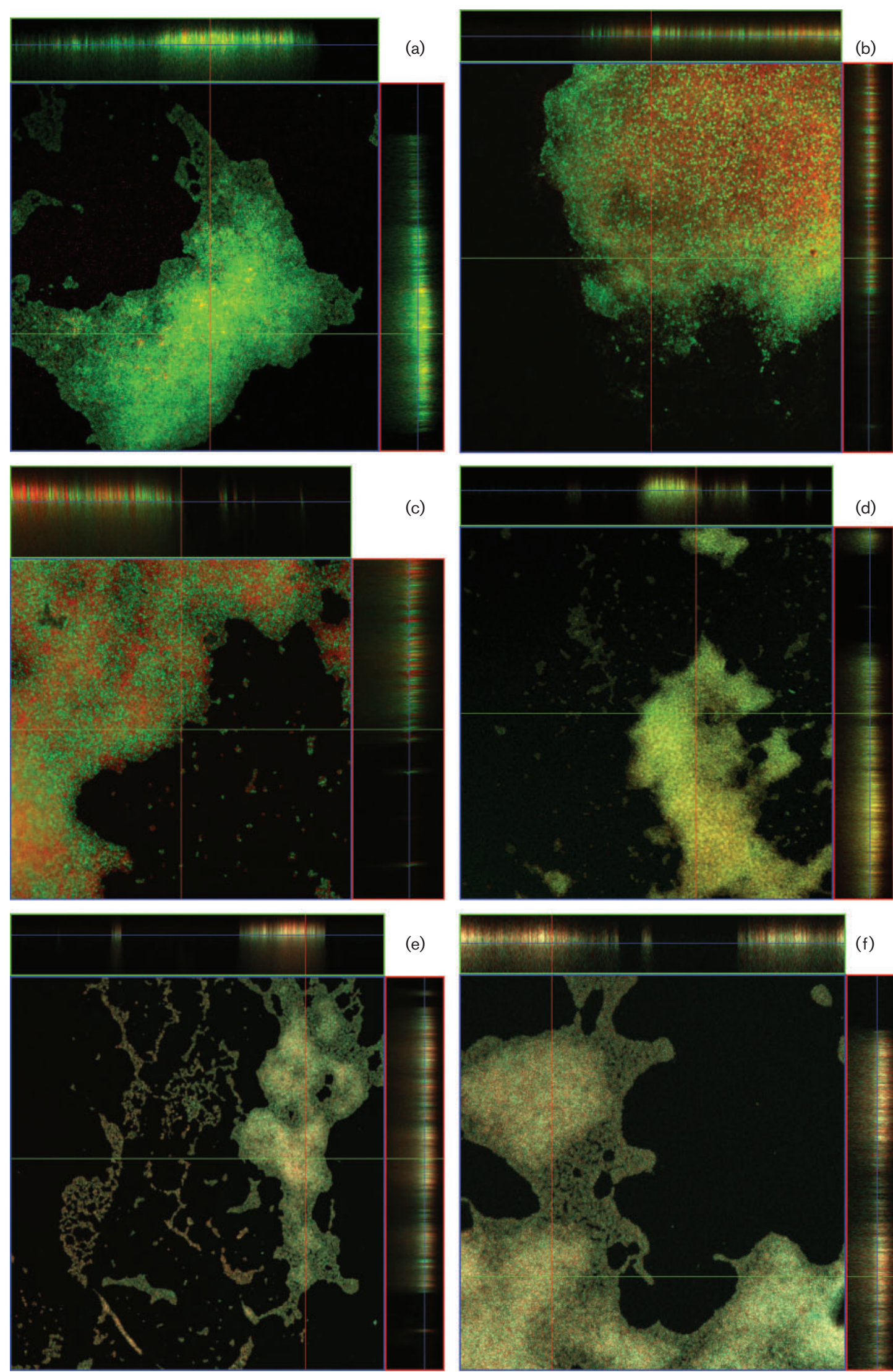
out under static conditions and antimicrobial agents were prepared in $0.9 \%$ saline. However, our biofilm model does differ from clinical biofilms. We used polystyrene as the biofilm platform, whereas catheters are mostly manufactured from polyurethane and Teflon. In addition, we prepared biofilms with inocula of $10^{7}$ c.f.u. $\mathrm{ml}^{-1}$, in contrast to the clinical situation, where catheters are generally seeded with bacteria at densities of 50-600 c.f.u. $\mathrm{ml}^{-1}$ (Shah et al., 2002). We chose polystyrene because it is commonly available in microbiology laboratories and has been widely used for biofilm studies. The initial inoculum of $10^{7}$ c.f.u. $\mathrm{ml}^{-1}$ was adopted for our study as this density has been accepted as a standard inoculum for in vitro biofilm formation (Deighton et al., 2001).

As we anticipated that agent carryover could be a problem in this study, which used antimicrobial agents at high concentrations, resin beads (Amberlite XAD-16) were used to remove any antimicrobial agents that remained after treatment and washing of biofilms. Although there are limited data about the efficacy of Amberlite XAD-16 in removal of antibiotics, the structurally similar but functionally weaker Amberlite XAD-4 efficiently reduces carryover of aminoglycosides, penicillin, macrolides, ciprofloxacin and vancomycin from bacterial cultures (Hyatt et al., 1994; Zabinski et al., 1993). Moreover, these resin beads have no adverse effect on bacterial growth (Hyatt et al., 1994; Zabinski et al., 1993).

Two bacterial recovery methods have been widely used by biofilm researchers (Raad et al., 2007; Zimmerli et al., 1994). One method consists of dissociating the biofilms by scraping, vortexing or/and sonication, followed by plating the bacteria on recovery agar plates (dissociating/plating method). This quantitative method has low sensitivity in qualitatively detecting the remaining living biofilm cells (Raad et al., 2007). This could be due to the inability of the scraping or sonication method to completely dissociate biofilm cells or the failure to recover shocked cells by direct plating (Raad et al., 2007; Zimmerli et al., 1994). In biofilm studies, sonication is more commonly used to dissociate the biofilms grown at the air-liquid interface of plastic pegs or inserts, such as those formed by the Gram-negative bacillus Pseudomonas aeruginosa (Ceri et al., 1999). For the bottom-growing CoNS biofilms used in our study, there was poor efficiency and a large variation in cell release when sonication was used (unpublished data). Moreover, cross-contamination between wells frequently occurred when sonication was employed. In another method, the treated biofilms were incubated for an additional $24 \mathrm{~h}$ in fresh broth prior to dissociating and plating (broth incubation/dissociating/plating). Whilst this method is labour-intensive, it has been reported to have greater accuracy in qualitative determination of viable cells (Raad et al., 2007). The method that we used to recover the living cells in biofilms after antimicrobial treatment is a variation of the broth incubation/dissociating/plating method. An extended incubation period of $48 \mathrm{~h}$ was used instead of direct plating. We showed that our recovery method had the same accuracy as the broth incubation/dissociating/ plating method, but was less labour-intensive when dealing with ethanol.

In summary, in the present study, we have described the first in vitro model to compare the efficacy of ethanol and antibiotics as a CLS. This study showed that ethanol has a higher efficacy than most of the commonly used antibiotics. We also suggest that using $40 \%$ ethanol for $1 \mathrm{~h}$ is ideal as a CLS targeting CoNS biofilms. Additional in vivo studies are required to confirm the clinical utility of our findings. The efficacy of ethanol locks could also be confirmed on catheters with CoNS biofilms removed from patients with clinical infections.

\section{ACKNOWLEDGEMENTS}

This work was supported by an Australian Postgraduate Award to Y.Q. The authors wish to thank Michelle Thacker, University of Melbourne, for assistance with CLSM.

\section{REFERENCES}

Ackoundou-N'guessan, C., Heng, A.-E., Guenu, S., Charbonne, F., Traore, O., Deteix, P. \& Souweine, B. (2006). Ethanol lock solution as an adjunct treatment for preventing recurrent catheter-related sepsis - first case report in dialysis setting. Nephrol Dial Transplant 21, 3339-3340.

Alam, M., Sultana, M., Nair, G. B., Siddique, A. K., Hasan, N. A., Sack, R. B., Sack, D. A., Ahmed, K. U., Sadique, A. \& other authors (2007). Viable but nonculturable Vibrio cholerae $\mathrm{O} 1$ in biofilms in the aquatic environment and their role in cholera transmission. Proc Natl Acad Sci U S A 104, 17801-17806.

Allon, M. (2003). Prophylaxis against dialysis catheter-related bacteremia with a novel antimicrobial lock solution. Clin Infect Dis 36, 1539-1544.

Allon, M. (2004). Dialysis catheter-related bacteremia: treatment and prophylaxis. Am J Kidney Dis 44, 779-791.

Bailey, E., Berry, N. \& Cheesbrough, J. S. (2002). Antimicrobial lock therapy for catheter-related bacteraemia among patients on maintenance haemodialysis. J Antimicrob Chemother 50, 615-617.

Bastani, B., Minton, J. \& Islam, S. (2000). Insufficient penetration of systemic vancomycin into the PermCath lumen. Nephrol Dial Transplant 15, 1035-1037.

Benjamin, D. K., Jr, Miller, W., Garges, H., Benjamin, D. K., McKinney, R. E., Jr, Cotton, M., Fisher, R. G. \& Alexander, K. A. (2001). Bacteremia, central catheters, and neonates: when to pull the line. Pediatrics 107, 1272-1276.

Berney, M., Hammes, F., Bosshard, F., Weilenmann, H. U. \& Egli, T. (2007). Assessment and interpretation of bacterial viability by using the LIVE/DEAD BacLight Kit in combination with flow cytometry. Appl Environ Microbiol 73, 3283-3290.

Berrington, A. \& Gould, F. K. (2001). Use of antibiotic locks to treat colonized central venous catheters. J Antimicrob Chemother 48, 597-603.

Bradford, R., Abdul Manan, R., Daley, A. J., Pearce, C., Ramalingam, A., D'Mello, D., Mueller, Y., Uahwatanasakul, W., Qu, Y. \& other authors (2006). Coagulase-negative staphylococci in very-low-birth-weight infants: inability of genetic markers to distinguish invasive strains from blood culture contaminants. Eur J Clin Microbiol Infect Dis 25, 283-290. 
Castagnola, E., Moroni, C., Gandullia, P., Oddone, M., Peri, C., Casciaro, R. \& De Alessandri, A. (2006). Catheter lock and systemic infusion of linezolid for treatment of persistent Broviac catheterrelated staphylococcal bacteremia. Antimicrob Agents Chemother 50, 1120-1121.

Ceri, H., Olson, M. E., Stremick, C., Read, R. R., Morck, D. \& Buret, A. (1999). The Calgary Biofilm Device: new technology for rapid determination of antibiotic susceptibilities of bacterial biofilms. J Clin Microbiol 37, 1771-1776.

Christensen, G. D., Simpson, W. A., Younger, J. J., Baddour, L. M., Barrett, F. F., Melton, D. M. \& Beachey, E. H. (1985). Adherence of coagulase-negative staphylococci to plastic tissue culture plates: a quantitative model for the adherence of staphylococci to medical devices. J Clin Microbiol 22, 996-1006.

Curtin, J., Cormican, M., Fleming, G., Keelehan, J. \& Colleran, E. (2003). Linezolid compared with eperezolid, vancomycin, and gentamicin in an in vitro model of antimicrobial lock therapy for Staphylococcus epidermidis central venous catheter-related biofilm infections. Antimicrob Agents Chemother 47, 3145-3148.

Deighton, M. A., Capstick, J., Domalewski, E. \& van Nguyen, T. (2001). Methods for studying biofilms produced by Staphylococcus epidermidis. Methods Enzymol 336, 177-195.

Donlan, R. M. (2000). Role of biofilms in antimicrobial resistance. ASAIO J 46, S47-S52.

Dunne, W. M., Jr, Mason, E. O., Jr \& Kaplan, S. L. (1993). Diffusion of rifampin and vancomycin through a Staphylococcus epidermidis biofilm. Antimicrob Agents Chemother 37, 2522-2526.

Edmiston, C. E., Jr, Goheen, M. P., Seabrook, G. R., Johnson, C. P., Lewis, B. D., Brown, K. R. \& Towne, J. B. (2006). Impact of selective antimicrobial agents on staphylococcal adherence to biomedical devices. Am J Surg 192, 344-354.

Fernandez-Hidalgo, N., Almirante, B., Calleja, R., Ruiz, I., Planes, A. M., Rodriguez, D., Pigrau, C. \& Pahissa, A. (2006). Antibiotic-lock therapy for long-term intravascular catheter-related bacteraemia: results of an open, non-comparative study. J Antimicrob Chemother 57, 1172-1180.

Gagnon, R. F., Richards, G. K. \& Subang, R. (1993). Vancomycin therapy of experimental peritoneal catheter-associated infection (Staphylococcus epidermidis) in a mouse model. Perit Dial Int 13 (Suppl. 2), S310-S312.

Guedon, C., Nouvellon, M., Lalaude, O. \& Lerebours, E. (2002). Efficacy of antibiotic-lock technique with teicoplanin in Staphylococcus epidermidis catheter-related sepsis during long-term parenteral nutrition. JPEN J Parenter Enteral Nutr 26, 109-113.

Hyatt, J. M., Nix, D. E. \& Schentag, J. J. (1994). Pharmacokinetic and pharmacodynamic activities of ciprofloxacin against strains of Streptococcus pneumoniae, Staphylococcus aureus, and Pseudomonas aeruginosa for which MICs are similar. Antimicrob Agents Chemother 38, 2730-2737.

Kite, P., Eastwood, K., Sugden, S. \& Percival, S. L. (2004). Use of in vivo-generated biofilms from hemodialysis catheters to test the efficacy of a novel antimicrobial catheter lock for biofilm eradication in vitro. J Clin Microbiol 42, 3073-3076.

Klingenberg, C., Aarag, E., Ronnestad, A., Sollid, J. E., Abrahamsen, T. G., Kjeldsen, G. \& Flaegstad, T. (2005). Coagulase-negative staphylococcal sepsis in neonates. Association between antibiotic resistance, biofilm formation and the host inflammatory response. Pediatr Infect Dis J 24, 817-822.

Laird, J., Soutar, R. \& Butcher, I. (2005). Complications of the ethanol-lock technique in the treatment of central venous catheter sepsis. J Infect 51, 338.

Lee, J. Y., Ko, K. S., Peck, K. R., Oh, W. S. \& Song, J. H. (2006). In vitro evaluation of the antibiotic lock technique (ALT) for the treatment of catheter-related infections caused by staphylococci. J Antimicrob Chemother 57, 1110-1115.

Lewis, K. (2005). Persister cells and the riddle of biofilm survival. Biochemistry (Mosc) 70, 267-274.

Mermel, L. A., Farr, B. M., Sherertz, R. J., Raad, I. I., O'Grady, N., Harris, J. S. \& Craven, D. E. (2001). Guidelines for the management of intravascular catheter-related infections. Clin Infect Dis 32, 1249-1272.

Messing, B., Peitra-Cohen, S., Debure, A., Beliah, M. \& Bernier, J. J. (1988). Antibiotic-lock technique: a new approach to optimal therapy for catheter-related sepsis in home-parenteral nutrition patients. JPEN J Parenter Enteral Nutr 12, 185-189.

Metcalf, S. C., Chambers, S. T. \& Pithie, A. D. (2004). Use of ethanol locks to prevent recurrent central line sepsis. J Infect 49, 20-22.

Monzon, M., Oteiza, C., Leiva, J. \& Amorena, B. (2001). Synergy of different antibiotic combinations in biofilms of Staphylococcus epidermidis. J Antimicrob Chemother 48, 793-801.

O’Neill, A. J., Miller, K., Oliva, B. \& Chopra, I. (2004). Comparison of assays for detection of agents causing membrane damage in Staphylococcus aureus. J Antimicrob Chemother 54, 1127-1129.

Onland, W., Shin, C. E., Fustar, S., Rushing, T. \& Wong, W. Y. (2006). Ethanol-lock technique for persistent bacteremia of long-term intravascular devices in pediatric patients. Arch Pediatr Adolesc Med 160, 1049-1053.

Opilla, M. T., Kirby, D. F. \& Edmond, M. B. (2007). Use of ethanol lock therapy to reduce the incidence of catheter-related bloodstream infections in home parenteral nutrition patients. JPEN $J$ Parenter Enteral Nutr 31, 302-305.

Qin, Z., Lee, B., Yang, L., Zhang, J., Yang, X., Qu, D., Jiang, H. \& Molin, S. (2007a). Antimicrobial activities of YycG histidine kinase inhibitors against Staphylococcus epidermidis biofilms. FEMS Microbiol Lett 273, 149-156.

Qin, Z., Yang, X., Yang, L., Jiang, J., Ou, Y., Molin, S. \& Qu, D. (2007b). Formation and properties of in vitro biofilms of ica-negative Staphylococcus epidermidis clinical isolates. J Med Microbiol 56, 83-93.

Raad, I., Hanna, H., Dvorak, T., Chaiban, G. \& Hachem, R. (2007). Optimal antimicrobial catheter lock solution, using different combinations of minocycline, EDTA, and 25-percent ethanol, rapidly eradicates organisms embedded in biofilm. Antimicrob Agents Chemother 51, 78-83.

Saginur, R., Stdenis, M., Ferris, W., Aaron, S. D., Chan, F., Lee, C. \& Ramotar, K. (2006). Multiple combination bactericidal testing of staphylococcal biofilms from implant-associated infections. Antimicrob Agents Chemother 50, 55-61.

Shah, C. B., Mittelman, M. W., Costerton, J. W., Parenteau, S., Pelak, M., Arsenault, R. \& Mermel, L. A. (2002). Antimicrobial activity of a novel catheter lock solution. Antimicrob Agents Chemother 46, 1674-1679.

Shama, A., Patole, S. K. \& Whitehall, J. S. (2002). Intravenous rifampicin in neonates with persistent staphylococcal bacteraemia. Acta Paediatr 91, 670-673.

Sherertz, R. J., Boger, M. S., Collins, C. A., Mason, L. \& Raad, I. I. (2006). Comparative in vitro efficacies of various catheter lock solutions. Antimicrob Agents Chemother 50, 1865-1868.

Sissons, C. H., Wong, L. \& Cutress, T. W. (1996). Inhibition by ethanol of the growth of biofilm and dispersed microcosm dental plaques. Arch Oral Biol 41, 27-34.

Spoering, A. L. \& Lewis, K. (2001). Biofilms and planktonic cells of Pseudomonas aeruginosa have similar resistance to killing by antimicrobials. J Bacteriol 183, 6746-6751.

Stocks, S. M. (2004). Mechanism and use of the commercially available viability stain, BacLight. Cytometry A 61, 189-195. 
Stoll, B. J., Hansen, N., Fanaroff, A. A., Wright, L. L., Carlo, W. A., Ehrenkranz, R. A., Lemons, J. A., Donovan, E. F., Stark, A. R. \& other authors (2002). Late-onset sepsis in very low birth weight neonates: the experience of the NICHD Neonatal Research Network. Pediatrics 110, 285-291.

van de Wetering, M. D. \& van Woensel, J. B. (2007). Prophylactic antibiotics for preventing early central venous catheter Gram positive infections in oncology patients. Cancer Treat Rev 31, 186-196.

Villari, P., Sarnataro, C. \& lacuzio, L. (2000). Molecular epidemiology of Staphylococcus epidermidis in a neonatal intensive care unit over a three-year period. J Clin Microbiol 38, 1740-1746. von Eiff, C., Peters, G. \& Heilmann, C. (2002). Pathogenesis of infections due to coagulase-negative staphylococci. Lancet Infect Dis 2, 677-685.

Zabinski, R. A., Larsson, A. J., Walker, K. J., Gilliland, S. S. \& Rotschafer, J. C. (1993). Elimination of quinolone antibiotic carryover through use of antibiotic-removal beads. Antimicrob Agents Chemother 37, 1377-1379.

Zimmerli, W., Frei, R., Widmer, A. F. \& Rajacic, Z. (1994). Microbiological tests to predict treatment outcome in experimental device-related infections due to Staphylococcus aureus. J Antimicrob Chemother 33, 959-967. 